\title{
DE LAS COMPETENCIAS BÁSICAS A LAS COMPETENCIAS CLAVE: CONCEPTUALIZACIÓN DESDE LA EDUCACIÓN FÍSICA
}

\author{
Jorge Agustín Zapatero Ayuso \\ Universidad Complutense de Madrid \\ María Dolores González Rivera \\ Universidad de Alcalá \\ Antonio Campos Izquierdo \\ Universidad Politécnica de Madrid
}

\section{RESUMEN}

Las competencias clave han replanteado la manera de enfocar la enseñanza y los aprendizajes, que deben seleccionarse para la consecución de las mismas y ser orientados de manera funcional y práctica. En este contexto, el modelo competencial ha exigido distintos enfoques en la Educación Física, cuyo profesorado debe comprehender las competencias y abordar las mismas en su desempeño habitual. Sin embargo, los estudios han demostrado que este elemento curricular parece desconocido en los centros educativos y predomina la falta de formación. En consecuencia, este trabajo pretende ser una herramienta didáctica para el docente de Educación Física. Con esta finalidad, se conceptualiza sobre las competencias clave en la asignatura y el rol que desempeña la Educación Física para su desarrollo a través de un análisis normativo y de expertos.

Palabras Clave: competencias, Educación Física, programa educativo, formación docente.

\section{FROM BASIC COMPETENCIES TO KEY COMPETENCIES: CONCEPTUALIZATION FROM PHYSICAL EDUCATION}

\section{Abstract}

Key competencies have rethought the way we approach teaching and learning outcomes, which should be selected to achieve them and be oriented functional and practical way. In this context, the model based on competencies has required different approaches in Physical Education; the teacher must understand the competencies and develop them in his usual performance. However, research findings have demonstrated that this curricular element seems unknown in schools and dominates the lack of training. Consequently, this paper aims to be an educational tool for teaching Physical Education. To this objective, it is conceptualized on key competences in the subject and the role played by physical education for development through normative and expert analysis.

KeYwords: competencies, Physical Education, educational program, teacher training.

DOI: http://doi.org/10.25145/j.qurricul.2018.31.004

Revista Qurriculum, 31; junio 2018, pp. 77-95; ISSN: e-2530-8386 


\section{INTRODUCCIÓN}

Enseñar por competencias supone contribuir a su desarrollo con el fin de que el alumno sea capaz de aplicar sus aprendizajes para resolver situaciones complejas (Tejada, 2012). No obstante, el concepto de competencia es un término difuso, que se ha caracterizado por una pluralidad conceptual (Ellström y Kock, 2008; Illeris, 2008). La OCDE define la competencia como la capacidad para responder con éxito a exigencias complejas en un contexto particular, movilizando conocimientos, aptitudes cognitivas y prácticas, y componentes sociales y comportamentales como actitudes, emociones, valores y motivaciones (Rychen y Salganik, 2004). Por su parte, el Consejo Europeo (2006) entiende por competencia «una combinación de conocimientos, capacidades y actitudes adecuados para una determinada situación" (p. 13). Concretamente, las competencias básicas o clave reúnen tres criterios (Tiana, Moya y Luengo, 2011): están al alcance de todo el mundo y no son selectivas; son relevantes en una amplia gama de situaciones de vida y se asocian a prácticas sociales; contribuyen al «aprendizaje a lo largo de la vida» y pueden ser consideradas más instrumentales con respecto a otras más específicas. Para Martínez-López (2009) las competencias básicas representan una capacidad de respuesta idónea a situaciones diversas, son una combinación de conocimientos, habilidades y actitudes, deben adquirirse al finalizar la enseñanza obligatoria y se manifiestan en el desarrollo de procesos mentales complejos que permiten actuar en situaciones concretas y reales.

No obstante, ¿qué competencias son necesarias para ser un ciudadano activo, favorecer la cohesión social y la empleabilidad? Éste es un aspecto tan decisivo como la propia conceptualización del término (Sarramona, 2004). La definición y selección de competencias y del contenido de las mismas fue una preocupación para implementar modelos curriculares por competencias. Algunos organismos internacionales, como la OCDE, a través de su Proyecto DeSeCo (Rychen y Salganik, 2004), o el Consejo Europeo (2006), a través de las Recomendaciones del Parlamento Europeo y del Consejo sobre las competencias clave para el aprendizaje permanente, intentaron identificar las competencias necesarias para desempeñar en la sociedad del conocimiento. El proyecto DeSeCo identificó tres amplias categorías de competencias fundamentales para la vida: usar herramientas de manera interactiva, actuar de forma autónoma e interactuar en grupos heterogéneos. El Consejo Europeo (2006) concretó ocho competencias clave: comunicación en la lengua materna, comunicación en lenguas extranjeras, competencia matemática y competencias básicas en ciencias y tecnología, competencia digital, aprender a aprender, competencias interpersonales, interculturales y sociales y competencia cívica, espíritu de empresa y expresión cultural.

En este contexto de cambio, el sistema educativo español se sumó a la reforma competencial incorporando en la Ley Orgánica 2/2006, de 3 de mayo, de Educación (LOE), ocho competencias básicas. De este modo, España pretendía seguir los planteamientos del «aprendizaje a lo largo de la vida» (Consejo Europeo, 2006). Actualmente, la modificación planteada por la Ley Orgánica 8/2013, de 9 de diciembre, para la mejora de la calidad educativa (LOE-LOMCE) reformuló las ocho competencias básicas en siete competencias clave (tabla 1). No obstante, 
ambas propuestas responden a las orientaciones europeas, al mismo tiempo que la LOE-LOMCE ha refrendado el rol curricular de las competencias (Hortigüela, Abella y Pérez-Pueyo, 2015).

\begin{tabular}{|c|c|c|}
\hline \multicolumn{3}{|c|}{$\begin{array}{c}\text { TABLA 1. PROPUESTAS DE COMPETENCIAS CLAVE EN EL ÁMBITO EUROPEO } \\
\text { Y SU EVOLUCIÓN EN EL SISTEMA EDUCATIVO ESPAÑOL }\end{array}$} \\
\hline Propuesta del Consejo Europeo & Concreción LOE & MODIFICACIÓN LOMCE \\
\hline $\begin{array}{l}\text { 1. Comunicación en la lengua } \\
\text { materna }\end{array}$ & 1. Comunicación lingüística & 1. Comunicación lingüística \\
\hline $\begin{array}{l}\text { 3. Competencia matemática y com- } \\
\text { petencias básicas en ciencias } \\
\text { y tecnología }\end{array}$ & $\begin{array}{l}\text { 2. Matemática } \\
\text { 3. Conocimiento y la interacción } \\
\text { con el mundo físico }\end{array}$ & $\begin{array}{l}\text { 2. Competencia matemática y } \\
\text { competencias básicas } \\
\text { en ciencias y tecnología }\end{array}$ \\
\hline 4. Competencia digital & $\begin{array}{l}\text { 4. Tratamiento de la información } \\
\text { y competencia digital }\end{array}$ & 3. Competencia digital \\
\hline 5. Aprender a aprender & 5. Aprender a aprender & 4. Aprender a aprender \\
\hline $\begin{array}{l}\text { 6. Competencias interpersonales, } \\
\text { interculturales y sociales } \\
\text { y competencia cívica }\end{array}$ & 6. Social y ciudadana & 5. Sociales y cívicas \\
\hline 7. Espíritu de empresa & 7. Autonomía e iniciativa personal & $\begin{array}{l}\text { 6. Sentido de iniciativa } \\
\text { y espíritu emprendedor }\end{array}$ \\
\hline 8. Expresión cultural & 8. Cultural y artística & $\begin{array}{l}\text { 7. Conciencia y expresiones } \\
\text { culturales }\end{array}$ \\
\hline
\end{tabular}

Sin embargo, a pesar de que España ha sido uno de los países que mayor número de normas ha desarrollado en materia competencial (European Commission, ECAEA y Eurydice, 2012), los estudios han demostrado que éstas son unas desconocidas para el profesorado y el modelo competencial no llega a implementarse (Barrachina y Blasco, 2012; Caballero, 2013; Zapatero-Ayuso, González-Rivera y Campos-Izquierdo, 2012). Parece que la norma se percibe difusa y dificulta el proceso para abordar las competencias en los centros educativos, lo que lleva a que la normativa y las competencias se repitan en los documentos de centro o se concreten de forma superficial (Barrachina y Blasco, 2012; Caballero, 2013; Monarca y Rappoport, 2013; Hortigüela et al., 2015). Por tanto, podría ser de interés asesorar e informar al profesorado para que haga de las competencias elementos operativos en sus centros y aulas (Ramírez, 2015), y es que el profesorado es el agente principal para llevar a cabo la reforma competencial (Gordon et al., 2009). Partiendo de este planteamiento, con este trabajo se pretende ofrecer una herramienta didáctica para el profesorado de Educación Física (EF). Específicamente, el objetivo fue establecer unos descriptores que ayuden a comprehender las competencias y orientar cómo realizar la contribución en la EF, al mismo tiempo que se pretende impulsar la concreción competencial de forma inductiva desde la asignatura (Pérez-Pueyo, 2013). 


\section{PROCEDIMIENTO PARA LA CONCRECIÓN DE LAS COMPETENCIAS}

Para atender a los objetivos de este trabajo, se revisaron las orientaciones normativas surgidas en relación con las competencias básicas y clave en el sistema educativo español y en Europa (normativa LOE, LOE-LOMCE y sobre «aprendizaje a lo largo de la vida", respectivamente). Además, se profundizó sobre la contribución a las competencias en EF a través de una revisión bibliográfica (tabla 2). De este modo, se generó un modelo de descriptores inicial con el fin de orientar la contribución a las competencias clave en la asignatura. Este primer modelo fue revisado en una verificación intersubjetiva entre los participantes en este trabajo con el propósito de valorar la pertinencia de los descriptores y la adecuada redacción y comprensión de los mismos (Pérez-Serrano, 1994). Siguiendo a Pérez-Pueyo (2013) y utilizando una terminología competencial, el procedimiento seguido fue inductivo conceptualizando sobre las competencias desde una asignatura en particular, la EF. Ahora bien, para la aplicación práctica en los centros educativos, este paso de conceptualización desde una materia en cuestión debe ser sucedido por el debate y la reflexión colaborativa en los claustros, con el fin de alcanzar acuerdos y líneas de trabajo comunes que deriven en intervenciones educativas globales de acuerdo a planteamientos competenciales (Hong, 2013). En este análisis inductivo se obtuvieron los resultados que se presentan a continuación.

\section{LAS COMPETENCIAS CLAVE EN LA EDUCACIÓN FÍSICA}

Las competencias se definieron en varios descriptores para la EF (tabla 2). A continuación, se desarrollan estos descriptores analizando cada competencia en particular.

\section{TABLA 2. DESCRIPTORES DE LAS COMPETENCIAS CLAVE EN LA EDUCACIÓN FÍSICA}

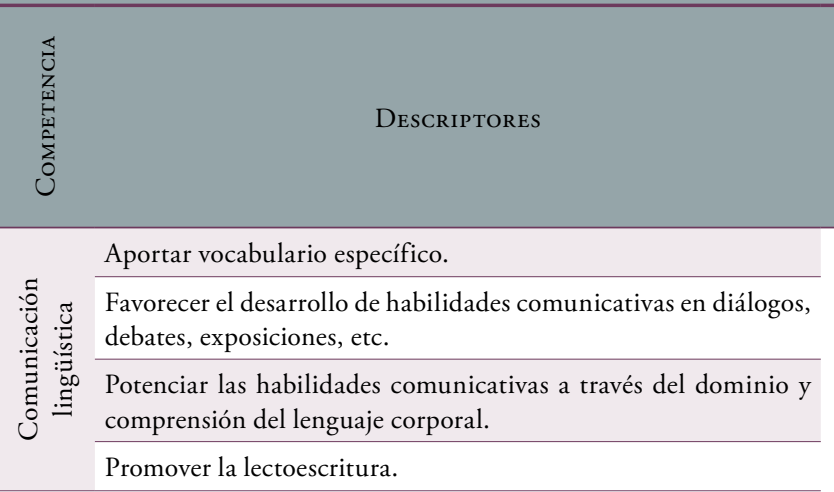

- Blázquez y Sebastiani (2009)

- Calahorro, Lara y Torres-Luque (2010)

- Cañabate, Zagalaz, Lara y Chacón (2011) - Consejo Europeo (2006) 


\begin{tabular}{|c|c|c|}
\hline \multirow{6}{*}{ 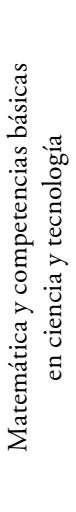 } & $\begin{array}{l}\text { Desarrollar aquellas cualidades físicas asociadas a la salud: resis- } \\
\text { tencia, fuerza resistencia y flexibilidad. }\end{array}$ & - Contreras y Cuevas (2011) \\
\hline & $\begin{array}{l}\text { Facilitar el análisis y crítica del impacto de la actividad física y el } \\
\text { deporte en el medio ambiente, promoviendo un uso responsable } \\
\text { del mismo. }\end{array}$ & $\begin{array}{l}\text { - Decreto } 23 / 2007 \\
\text { - Decreto } 57 / 2007\end{array}$ \\
\hline & Favorecer la interacción con el medio físico natural o semiartificial. & - Decreto 74/2007 \\
\hline & $\begin{array}{l}\text { Promover la actividad física y el deporte como medio para la } \\
\text { consecución y mantenimiento de una vida higiénica-saludable. }\end{array}$ & - Decreto 89/2014 \\
\hline & $\begin{array}{l}\text { Proporcionar y favorecer el uso de habilidades de cálculo para el } \\
\text { control/gestión de actividad física y deporte. }\end{array}$ & - Decreto $133 / 2007$ \\
\hline & $\begin{array}{l}\text { Procurar la aplicación de habilidades y herramientas de cálculo } \\
\text { para gestionar su actividad diaria favoreciendo el mantenimiento } \\
\text { de una vida saludable. }\end{array}$ & - Decreto $175 / 2007$ \\
\hline \multirow{3}{*}{$\frac{\pi}{0.00}$} & $\begin{array}{l}\text { Proporcionar herramientas de las Tecnologías de la Información } \\
\text { y la Comunicación (TIC) para acceder a información relevante } \\
\text { de actividad física y deporte. }\end{array}$ & $\begin{array}{l}\text { - Direction générale de l'en- } \\
\text { seignement scolaire (2006) }\end{array}$ \\
\hline & $\begin{array}{l}\text { Proporcionar herramientas TIC para elegir y gestionar su propia } \\
\text { práctica de actividad física y deporte. }\end{array}$ & - Giráldez (2007) \\
\hline & Usar TIC como apoyo a la docencia dentro y/o fuera del aula. & - López-Pacheco (2010) \\
\hline \multirow{3}{*}{ 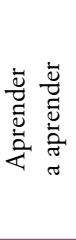 } & $\begin{array}{l}\text { Orientar la actividad física y el deporte para la aplicación en el } \\
\text { tiempo libre de los alumnos. }\end{array}$ & - Martín y Monereo (2007) \\
\hline & $\begin{array}{l}\text { Permitir que el alumno conozca sus capacidades: fortalezas y } \\
\text { debilidades. }\end{array}$ & - Monereo (2005) \\
\hline & $\begin{array}{l}\text { Proporcionar técnicas de estudio y favorece que el alumno gestione } \\
\text { su aprendizaje. }\end{array}$ & - Moya (2008) \\
\hline \multirow{4}{*}{ 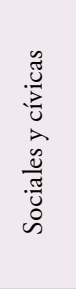 } & Favorecer la cooperación y el trabajo en equipo. & - Muñoz-Díaz (2010) \\
\hline & Fomentar la reflexión y el pensamiento crítico. & - Orden ECD/65/2015 \\
\hline & $\begin{array}{l}\text { Relacionar la actividad física con fenómenos sociohistóricos: } \\
\text { globalización, consumismo, racismo, dopaje, medios de comu- } \\
\text { nicación, etc. }\end{array}$ & $\begin{array}{l}\text { - Quintero, Jiménez-Jiménez y } \\
\text { Area (2016) }\end{array}$ \\
\hline & $\begin{array}{l}\text { Usar procedimientos democráticos: negociación, votación, de- } \\
\text { bate, etc. }\end{array}$ & - Real Decreto 1513/2006 \\
\hline \multirow{4}{*}{ 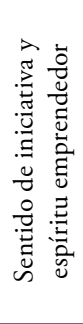 } & $\begin{array}{l}\text { Favorecer el espíritu emprendedor, la autocrítica, la autosuperación } \\
\text { y/o la actitud positiva para la mejora de sus capacidades físicas o } \\
\text { la ejecución de sus habilidades motrices. }\end{array}$ & - Real Decreto 1631/2006 \\
\hline & $\begin{array}{l}\text { Incitar a la proposición de cambios acerca de elementos curricula- } \\
\text { res: contenidos, objetivos, criterios, metodologías, etc. }\end{array}$ & - Real Decreto 126/2014 \\
\hline & $\begin{array}{l}\text { Permitir la participación en proyectos o jornadas de acuerdo a las } \\
\text { necesidades de actividad física y/o deporte del alumnado. }\end{array}$ & - Rojano (2010) \\
\hline & Permitir que el alumno diseñe, gestione y/o guíe actividades & - Salmerón (2010a, 2010b) \\
\hline \multirow{2}{*}{ 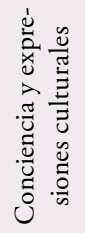 } & $\begin{array}{l}\text { Promover el conocimiento, la aceptación y la actitud abierta } \\
\text { hacia prácticas de actividades físico-deportivas multiculturales, } \\
\text { autóctonas o extranjeras. }\end{array}$ & - Santos y Martínez (2010) \\
\hline & $\begin{array}{l}\text { Promover la expresión creativa, la imaginación y la adquisición } \\
\text { de habilidades perceptivas, de sensibilidad y el sentido estético. }\end{array}$ & - Ureña (2010) \\
\hline
\end{tabular}




\subsection{COMPETENCIA EN COMUNICACIÓN LINGÜÍSTICA}

Para el Consejo Europeo (2006), la comunicación lingüística en la lengua materna se define como la habilidad para expresar conceptos, pensamientos, hechos y opiniones de forma oral y escrita. Por tanto, incide sobre las habilidades de escucha, habla, lectura y escritura. Estas habilidades sirven para interactuar lingüísticamente en contextos sociales y culturales como la educación, la vida personal, profesional o en el ámbito de ocio y recreación. En la misma línea, Calahorro et al. (2010) apuntan que esta competencia tiene implicaciones en la comunicación oral y escrita, la representación e interpretación de la realidad, la construcción y comunicación del conocimiento y la autorregulación del pensamiento, las emociones y la conducta.

Concretamente, la EF puede reforzar el aprendizaje de otras lenguas, favorecer la transmisión de afectos, estados de ánimo y señales con el cuerpo y permite favorecer la comunicación gestual, la respiración y, en general, las técnicas comunicativas de dominio corporal (Ureña, 2010). Al respecto, esta competencia no se limita a la adquisición de habilidades de exposición, comprensión oral o escrita en diferentes contextos, sino que, además, incluye el desarrollo de elementos expresivos. La motricidad facilita la comunicación y es un canal importante para potenciarla y facilitar la expresión de emociones, sentimientos o ideas a través del gesto, la postura o el movimiento. Al respecto, la expresión corporal puede ser un elemento de apoyo que favorezca un desempeño comunicativo más idóneo (López-Pacheco, 2010). Además, el carácter instrumental de esta competencia favorece que su contribución pueda desarrollarse desde diversos contenidos, al mismo tiempo que la organización de dinámicas en las que los alumnos deban expresarse e interactuar puede potenciar su adquisición. Desde un punto de vista estrictamente motriz, la materia puede potenciar esta competencia a través del control de la respiración y del dominio del lenguaje corporal.

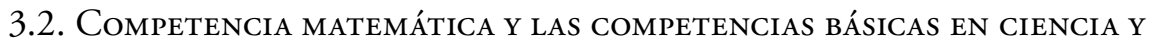 TECNOLOGÍA}

Esta competencia se define en dos grandes dimensiones: matemática, y ciencia y tecnología. La competencia matemática hace referencia al conocimiento y aplicación del razonamiento matemático para la resolución de conflictos. Se vincula a la movilización de modos matemáticos de pensamiento (lógico y espacial) y representación (fórmulas, modelos, construcciones, gráficos y diagramas). La competencia científica y tecnológica alude a la capacidad y la voluntad de utilizar los conocimientos y metodología para explicar la naturaleza y los cambios causados por el ser humano, para plantear cuestiones y obtener respuestas en base a pruebas (Consejo Europeo, 2006).

En cuanto a la competencia matemática, López-Pacheco (2010) considera que el dominio de esta competencia conlleva la definición y comprensión de términos, conceptos, leyes, teorías y modelos relacionados con la EF. Asimismo, esta competencia incluye la identificación y uso de estrategias para utilizar razonamientos, símbolos y fórmulas, con el fin de codificar e interpretar la realidad y abordar 
numerosas situaciones cotidianas relacionadas con la propia salud y la calidad de vida y su mejora a través de la práctica de actividad física y las técnicas de relajación. Ureña (2010) concreta la contribución a esta competencia atendiendo a tres aspectos:

- Empleo de fórmulas para calcular índice de masa corporal, umbral aeróbico, Test de Ruffier, cálculo de tiempos, pulsaciones, distancias, estructuración espacial, la estructuración espaciotemporal, etc.

- Elaboración e interpretación de gráficos relacionados con una vida saludable.

- Elaboración e interpretación de perfiles topográficos, rutómetros, mapas, etc., relacionados con las actividades en el medio natural.

En consecuencia, esta competencia puede vincularse con el uso y cálculo de parámetros de medida de la condición física o con el dibujo e interpretación de gráficos y mapas como elementos ligados a determinadas prácticas físicas. Además, de forma más vinculada a la competencia motriz, esta competencia mantiene una relación con la capacidad de estructuración temporal y espacial y los conceptos derivados de la misma.

En cuanto a la competencia en ciencia y tecnología, ésta parece fundamentada en la competencia en el conocimiento y la interacción con el mundo físico. Analizando ambas desde el punto de vista de la EF, se pueden identificar dos grandes ámbitos a los que contribuir: la promoción de la práctica física para una vida saludable y la impulsión del contacto y conservación del medio natural a través de la actividad física y el deporte. En relación con el primer ámbito, la introducción a la EF en los desarrollos curriculares LOE-LOMCE resalta el carácter prioritario de desarrollar competencias básicas en materia de salud, junto con la competencia motriz, para alcanzar el máximo bienestar físico, mental y social. Asimismo, esta competencia proporciona conocimientos sobre determinados hábitos saludables y aporta criterios para el mantenimiento y mejora de cualidades físicas (Ureña, 2010). En relación con el segundo ámbito, el uso del medio natural como recurso en la EF posibilita que el alumno interactúe con él de forma responsable, analizando las consecuencias de las acciones humanas y favoreciendo una práctica física sostenible y respetuosa con el medio (Santos y Martínez, 2011). Siguiendo a estos autores, esta competencia impulsa el análisis y conocimiento del medio para mejorar la interacción naturaleza-ser humano, desarrollando la capacidad y la disposición para lograr una vida saludable en un entorno también saludable. Ampliando esta visión, Calahorro et al. (2010) interpretan esta competencia como «la habilidad para la comprensión de sucesos, la predicción de las consecuencias y la actividad sobre el estado de personas y la sostenibilidad ambiental» (p. 34). Así, esta competencia tiene implicaciones en los aspectos generados por la acción humana para la mejora y la preservación de las condiciones de vida propias y colectivas. En consecuencia, la EF podría contribuir a esta competencia proporcionando los recursos necesarios para un adecuado desenvolvimiento de los individuos en el medio en que viven. Este hecho implica favorecer una capacidad funcional mínima para garantizar una proyección corporal del individuo sobre el medio y el dominio de esa interacción (Contreras y Cuevas, 2011). 


\subsection{Competencia digital}

La competencia digital entrańa el uso seguro y crítico de las tecnologías en la sociedad de la información con fines laborales, de ocio o comunicación. Se sustenta en el uso de ordenadores e Internet "para obtener, evaluar, almacenar, producir, presentar e intercambiar información y comunicarse y participar en redes de colaboración»(Consejo Europeo, 2006, p. 15).

Concretamente en la EF, esta competencia tiene que ver con el uso de las tecnologías de la información y la comunicación para la ampliación de conocimientos relativos a la asignatura y, más específicamente, con la práctica saludable de actividad física (Ureña, 2010). Además, el desarrollo de esta competencia clave exige un desempeño acorde con una "ciberciudadanía" segura y ejercida con una responsabilidad ética (Quintero et al., 2016). Para su consecución se requiere del tratamiento de cinco dimensiones (López-Pacheco, 2010; Quintero et al., 2016):

- Cognitiva-intelectual: movilizar recursos para encontrar, seleccionar y analizar información procedente de diversas fuentes en soporte digital (Youtube o Google).

- Instrumental: proceder a la síntesis, elaboración y comunicación de información principalmente a través de las tecnologías de transmisión (presentaciones, procesadores de textos, etc.).

- Sociocomunicativa: ser capaz de expresarse, comunicarse y trabajar colaborativamente en entornos virtuales.

- Axiológica: adquirir e interiorizar principios y valores éticos y democráticos de actuación en la Red.

- Emocional: control y regulación de sentimientos y emociones en las interacciones que se producen con las tecnologías de interacción virtuales.

En relación con la dimensión cognitivo-intelectual, en la actividad física y el deporte, las tecnologías de la información y la comunicación no sólo han estado ligadas al acceso y gestión de la información. Se han diseñado variados software y hardware destinados al conocimiento y a la gestión de la propia actividad física y el deporte. Algunos de estos sistemas son costosos, como las plataformas de fuerzas. Sin embargo, otros pueden ser de un coste asumible por un Departamento de EF, como la videocámara, teléfonos móviles o los pulsómetros. En el caso concreto de los teléfonos móviles, accesibles también a determinados grupos de alumnos, incorporan aplicaciones propias de la EF y con cierto potencial educativo, como en el caso de Anatomy 4d o Cooper Vo2 Max Test (Quintero et al., 2016). El empleo de estas herramientas en el aula parece incentivar al alumno y constituyen una posible aportación de la materia al desarrollo de la competencia digital (Rojano, 2010).

En cuanto a la dimensión instrumental, la colaboración y el trabajo en Internet puede contribuir a la resolución de problemas, siendo los alumnos aquéllos que indaguen sobre los mismos y construyan su propio aprendizaje y el contenido de la EF. Así, Quintero et al. (2016) presentan algunos recursos destinados a la resolución 
de problemas, como los códigos $\mathrm{QR}$, o la creación de contenidos, como los recursos de expresión (Prezi o Emaze), infografías (Glogster) o podcast (Audacity).

Desde el punto de vista social y ético, la revolución tecnológica ha modificado la comunicación en la actual sociedad del conocimiento (Martínez-López, 2009). En este sentido, el profesorado puede utilizar las herramientas de comunicación en red (por ejemplo, correos electrónicos, webquest, Blogger, Dropbox o Google Drive) para transmitir e intercambiar información con los alumnos y generar entornos virtuales de conocimiento sobre actividad física y deporte (Quintero et al., 2016). De hecho, Internet parece ser un espacio idóneo para el desarrollo de las competencias. En este espacio virtual los alumnos pasan gran cantidad de horas. El hecho de utilizar las herramientas tecnológicas puede facilitar el contacto con los alumnos y potenciar el rol de «docente facilitador» enseñando a sus alumnos a comunicarse, a participar con responsabilidad en la vida pública y a colaborar con otros a través de Internet (Monereo, 2005).

La revolución tecnológica ha calado hondo y la actividad física y el deporte se han apoyado en esta revolución para avanzar como ciencia. Estos cambios han facilitado la construcción y transmisión del conocimiento potenciando el crecimiento de las ciencias de la actividad física y el deporte. Las tecnologías de la información y la comunicación han proporcionado y exigido nuevas herramientas de comunicación a la docencia. Además, los desarrollos curriculares de la LOE-LOMCE han incluido las tecnologías de la información y la comunicación como contenido propio de la materia (Decreto 89/2014, p. 74). En consecuencia, los profesores de EF pueden y deben contribuir a esta competencia a través de sus contenidos, del uso de herramientas para la gestión y transmisión de la información y de la comunicación en Red entre docentes y alumnos. Por tanto, la planificación coherente y rigurosa de «e-actividades» se convierte en una práctica docente necesaria y decisiva para el desarrollo de la competencia digital (Quintero et al., 2016).

\subsection{Aprender A APRENDER}

«Aprender a aprender es la habilidad para iniciar el aprendizaje y persistir en él, para organizar su propio aprendizaje y gestionar el tiempo y la información eficazmente, ya sea individualmente o en grupos. Esta competencia conlleva ser consciente del propio proceso de aprendizaje y de las necesidades de aprendizaje de cada uno, determinar las oportunidades disponibles y ser capaz de superar los obstáculos con el fin de culminar el aprendizaje con éxito» (Consejo Europeo, 2006, p. 16).

A través de la práctica el alumno aprende a conocer cuáles son sus capacidades y a partir de ahí puede plantearse objetivos para mejorar éstas con esfuerzo y perseverancia (Ureńa, 2010). Por tanto, la EF puede favorecer el autoconocimiento y la competencia motriz y académica autopercibida, generando confianza en uno mismo para desarrollar el gusto y la motivación por continuar aprendiendo (Contreras y Cuevas, 2011). Para la consecución de este fin, es necesario adquirir herramientas y recursos que permitan controlar adecuadamente la práctica física para regular el aprendizaje de habilidades deportivas o desarrollar las capacidades físicas (Calahorro et al., 2010). 
Asimismo, la EF contribuye a esta competencia a través de la educación para el ocio y la ocupación del tiempo libre del alumno. En ese sentido, los contenidos de la materia pueden ser orientados a la adquisición de una práctica regular saludable que ocupe su tiempo libre. Por ejemplo, las actividades en el medio natural son un aliciente para la ocupación activa, constructiva y autónoma del tiempo de ocio de los jóvenes (Santos y Martínez, 2011).

No obstante, no se debe obviar el papel de la intervención docente en la contribución a esta competencia. Enseñar por competencias no sólo se preocupa por el qué enseñar, sino también por cómo se enseña, hecho que tiene una repercusión en la metodología y evaluación por competencias (Tejada, 2012; Méndez-Giménez, Sierra y Mañana, 2013). Al respecto, los modelos de evaluación y la metodología empleada en la enseñanza por competencias son elementos a través de los cuales contribuir a esta competencia. De hecho, para Martín y Monereo (2007) el desarrollo de esta competencia depende del modo en que los alumnos se acerquen al conocimiento. Por tanto, la participación del alumno en las evaluaciones y la metodología pueden ser factores que impulsen la contribución en la EF.

\subsection{COMPETENCIAS SOCIALES y CÍviCAS}

Estas competencias adquieren una relevancia notable en el marco normativo LOE-LOMCE. En su preámbulo (apartado XIV) se consideran esenciales para el desempeño la ciudadanía activa. Este apartado hace referencia a las Recomendaciones del Parlamento Europeo y del Consejo de 18 de diciembre de 2006 y aborda la necesidad de incluir una educación cívica y constitucional transversalmente en todas las materias. El Consejo Europeo (2006) afirma que esta competencia incluye todas las formas de comportamiento que preparan para participar constructivamente en la vida social y profesional y, en su caso, resolver conflictos. La EF plantea situaciones favorables para la adquisición de esta competencia desde cualquiera de sus contenidos (Contreras y Cuevas, 2011), al mismo tiempo que la metodología y la organización de sus sesiones puede propiciar la interacción social frecuente.

En relación con sus contenidos, el desarrollo de juegos y deportes de cooperación, con o sin oposición, parece ser especialmente favorable para fomentar la colaboración, la participación y el desarrollo de actividades dirigidas hacia la solidaridad, la cooperación y la no discriminación. Las tareas diseñadas para estos contenidos se relacionan con la convivencia, la participación y el conocimiento de la realidad social (Contreras y Cuevas, 2011). Además, la expresión corporal puede ocupar un lugar relevante, pues favorece las relaciones interpersonales, las cuales requieren de comunicación, lenguaje no verbal, expresión de ideas y sentimientos, empatía, escucha, confianza en los demás y colaboración en tareas conjuntas. En este sentido, la expresión corporal tiene mucho que aportar a las competencias sociales y cívicas (Blázquez y Sebastiani, 2009). Asimismo, la puesta en práctica de actividades motrices en situaciones de adaptación al entorno físico (Real Decreto 126/2014) conlleva la construcción de un código ético de actuación respetuosa ante el medio. Las actividades en el medio natural incentivan a la participación, la implicación del 
alumno y el desarrollo de habilidades sociales respetando el entorno natural (Santos y Martínez, 2011). Además, los contenidos sobre acondicionamiento físico y salud, reflejados de forma constante en el currículo LOE-LOMCE, agrupan aspectos relativos a la salud física, hábitos de actividad física y práctica deportiva regular, que inciden en el desarrollo de capacidades físicas para la mejora de la calidad de vida del alumno y el empleo constructivo de su tiempo libre. Esto se vincula directamente con la dimensión de participación y ciudadanía activa de esta competencia (Contreras y Cuevas, 2011). En esta línea, Ureña (2010) indica que la educación para la prevención de comportamientos de riesgo y la promoción de actividad física saludable es una prioridad para la EF.

En cuanto a la metodología de enseñanza en la EF, Contreras y Cuevas (2011) consideran que la EF puede ser una de las materias que más facilitan la socialización. Su didáctica específica ha indagado acerca de aspectos como la organización de los alumnos, la metodología, el desarrollo de proyectos o la puesta en práctica de juegos cooperativos (Delgado-Noguera, 1991). Por ende, esta materia puede contribuir a la adquisición de esta competencia a través de la participación del alumno en las sesiones, la colaboración responsable, la cooperación y el trabajo en equipo.

\subsection{Sentido De INICIATIVA Y ESPÍRITU EMPRENDEDOR}

Para el Consejo Europeo (2006) esta competencia se identifica con la habilidad de transformar las ideas en actos. Se vincula a la creatividad e innovación, así como con la habilidad para planificar y gestionar proyectos siguiendo objetivos. Esta competencia conlleva ser conscientes del contexto en el que se desarrolla la intervención del sujeto para aprovechar oportunidades de vida. Lo cual debe atender a una concienciación sobre los valores éticos y una adecuada gobernanza.

De acuerdo con la literatura sobre competencias en EF, parece que esta competencia se desarrolla a través de la gestión de factores organizacionales y evaluativos y del diseño de tareas. Se trata de una competencia muy transversal que no parece mantener una vinculación directa con contenidos específicos. Por tanto, los momentos desde los que contribuir a esta competencia se diversifican. Ureña (2010) organiza estas aportaciones en tres ámbitos: interacción en el aula, metodología seleccionada y exigencias y retos de las tareas (tabla 3).

\begin{tabular}{|c|c|c|}
\hline INTERACCIÓN EN EL AULA & Metodología SELECCIONADA & EXIGENCIAS Y RETOS DE LAS TAREAS \\
\hline $\begin{array}{l}\text { Clima motivacional. } \\
\text { Cooperación y participación de } \\
\text { los alumnos dentro y fuera del } \\
\text { aula. } \\
\text { Autogestión y cogestión de las } \\
\text { sesiones y actividades comple- } \\
\text { mentarias y extraescolares. }\end{array}$ & $\begin{array}{l}\text { Selección de propuestas más } \\
\text { activas e individualizadas que } \\
\text { favorezcan la autonomía e } \\
\text { iniciativa. }\end{array}$ & $\begin{array}{l}\text { Mejora de la condición física y } \\
\text { desarrollo de las habilidades motrices } \\
\text { desde su nivel inicial con responsabi- } \\
\text { lidad, autonomía e iniciativa. } \\
\text { Autosuperación y perseverancia } \\
\text { para la mejora de sus capacidades y } \\
\text { habilidades motrices. }\end{array}$ \\
\hline
\end{tabular}


Además, Contreras y Cuevas (2011) añaden que esta competencia puede ser desarrollada a través de la deliberación y la discusión de dilemas morales. En este sentido, se trata de plantear situaciones acontecidas o inventadas en el deporte, de modo que se tenga que discutir o reflexionar sobre estas situaciones individualmente $o$ en grupo. El diálogo y la reflexión exigen iniciativa personal y espíritu emprendedor.

Siguiendo estos planteamientos, esta competencia debe buscar la mejora de la iniciativa, la responsabilidad, el trabajo autónomo, la autoconfianza y la empatía a través del conocimiento de sus capacidades físicas, reflexionando sobre su aprendizaje y su nivel y adquiriendo e implementando estrategias para desarrollar sus capacidades físicas (Cañabate et al., 2011). En esta línea, las estrategias evaluativas pueden contribuir a la adquisición de esta competencia. El hecho de impulsar la coevaluación, autoevaluación y la valoración constante del trabajo del alumno en la asignatura parecen aspectos necesarios para que el alumno valore la adecuación y pertinencia de sus iniciativas.

\subsection{CoNCIENCIA Y EXPRESIONES CULTURALES}

El Consejo Europeo (2006) define esta competencia como la «apreciación de la importancia de la expresión creativa de ideas, experiencias y emociones a través de distintos medios, incluida la música, las artes escénicas, la literatura y las artes plásticas». Esta competencia pretende el aprendizaje de conocimientos, habilidades y actitudes culturales y artísticas básicas y su aplicación en contextos cotidianos. Concretamente en la EF, se pueden diferenciar dos dimensiones: conocimiento y valoración del patrimonio histórico-cultural de la actividad física y el deporte y la expresión artística y creativa en actividades de índole rítmica y expresiva. En cuanto al patrimonio artístico y cultural, la EF puede favorecer el conocimiento y la valoración de manifestaciones artísticas donde el cuerpo y el movimiento son un recurso muy importante. En este sentido, se puede transmitir el conocimiento, comprensión y valoración de las manifestaciones culturales de la motricidad humana como el deporte, los juegos populares, el teatro o la danza. En cuanto al ámbito artístico expresivo, la EF contribuye al conocimiento de lenguajes artísticos del cuerpo y su movimiento para desarrollar la expresión creativa. Se vincula a actividades como los cuentos motores, danzas, mimos o el teatro de sombras (Contreras y Cuevas, 2011). Para Giráldez (2007) se identifican tres ejes en el trabajo de esta competencia:

- El desarrollo de habilidades perceptivo-motrices.

- La participación en manifestaciones culturales como deportes, juegos tradicionales, la danza o el teatro.

- El conocimiento de manifestaciones lúdicas, expresivas y deportivas de otras culturas.

Siguiendo este planteamiento, la competencia conciencia y expresiones culturales puede ser trabajada en la EF a través de contenidos relativos a la expresión corporal y los juegos y deportes (Contreras y Cuevas, 2011). Al respecto, Blázquez 
y Sebastiani (2009) proponen actividades concretas como la organización y análisis de prácticas de actividad física representativas de determinadas épocas, como las Olimpiadas, la realización de danzas y bailes o la representación de obras teatrales literarias o de recreación de otros momentos históricos.

\section{CONSIDERACIONES FINALES}

En este trabajo se ha conceptualizado sobre el modo en que la EF puede contribuir a la adquisición de las competencias a través de sus contenidos y la intervención docente (metodología y evaluación). Se han podido definir unos descriptores o líneas globales de contribución a las competencias, al mismo tiempo que profundizar sobre ellos y presentar algunas estrategias de actuación para contribuir a las competencias clave desde la EF. Concretamente, se ha explicado cómo determinadas competencias se vinculan con determinados contenidos de la EF; por ejemplo, la competencia en comunicación lingüística con el lenguaje corporal, o la conciencia y expresiones culturales con el patrimonio artístico y cultural de la motricidad, como la danza, la dramatización o el propio juego y los deportes. Ahora bien, las competencias trascienden cualquier lógica disciplinar y no se debe adoptar una visión reduccionista (Bolívar, 2010). En este sentido, el análisis conceptual de este trabajo parece sugerir que un profesor creativo puede multiplicar los momentos para contribuir a las competencias en su desempeño profesional, aprovechando, no sólo los contenidos, sino también los recursos didácticos, la metodología y/o la evaluación en EF. De hecho, algunas metodologías propias de la asignatura, como los cuentos motores o los estilos de enseńanza participativos (Delgado-Noguera, 1991), suponen una contribución metodológica a las competencias, más allá de sus propios contenidos. Por tanto, la EF, apoyada en estos ámbitos de actuación y en colaboración con el resto de materias, parece contribuir a la adquisición de todas las competencias, proporcionando conocimientos, habilidades y actitudes a través de la vivencia de múltiples y variadas experiencias motrices, que impulsen también el desarrollo de la competencia motriz de sus alumnos. Al respecto y de acuerdo con el análisis realizado sobre cada competencia y sus descriptores, la tabla 4 sintetiza las estrategias más relevantes para contribuir a las competencias clave desde la EF mediante su metodología, sus recursos y/o evaluación.

\begin{tabular}{lll}
\hline \multicolumn{3}{c}{ TABLA 4. ESTRATEGIAS PARA CONTRIBUIR A LAS COMPETENCIAS } \\
CLAVE EN EDUCACIÓN FÍSICA
\end{tabular}


Empleo de gráficos para la representación del nivel de condición física o de habilidad motriz.

Matemática y competencias básicas en ciencia y tecnología
Empleo de fórmulas para la obtención de parámetros vinculados a la condición física (frecuencia cardiaca o índice de masa corporal). Elaboración de mapas y otros recursos topográficos para las actividades en el medio natural. Desarrollo de actividades complementarias y extraescolares en el medio natural.
Interpretación de gráficos sobre el nivel de condición física.

Aplicación de fórmulas para la valoración de su nivel de condición física.

Análisis del impacto de la actividad física en la preservación y cuidado del medio natural.
Uso de recursos digitales para el acceso y gestión de información (Youtube, Google, Pinterest).

Recursos sobre contenidos propios de la EF, como aplicaciones de teléfonos móviles (Anatomy $4 \mathrm{~d}$ o Cooper Vo2 Max Test Lite).

Digital Aprendizaje cooperativo y mediante resolución de problemas con recursos digitales (códigos QR, Dropbox o Google Drive).

Elaboración de "e-actividades" con wikis, webquest o ejercicios multimedia.

Propuesta de actividades con una orientación funcional y que sean enfocadas hacia la aplica-

Aprender a ción en el tiempo libre del alumnado.

aprender

Uso de metodologías que proporcionen protagonismo al alumno y garanticen su autonomía y toma de decisiones.
Empleo de instrumentos de evaluación como «e-portafolios» o test en línea.
Implicación de los alumnos en la evaluación de su competencia motriz como elemento de apoyo para guiar su aprendizaje (autoevaluaciones y coevaluaciones).

Desarrollo de juegos y tareas de cooperación y cooperación-oposición.

Sociales y cívicas ción, contacto entre compañeros y el trabajo en equipo.

Planteamiento transversal desde todos los contenidos de la EF.

Incluir al alumno en las decisiones del aula. Mantenimiento de un adecuado clima moti-

Sentido de iniciativa y espíritu emprendedor

\section{vacional.}

Usar metodologías que garanticen la toma de decisiones del alumno, impulsando la autogestión y cogestión de las sesiones.

Planteamiento transversal desde todos los contenidos de la EF.

Planteamiento de actividades que impulsen la expresión creativa (cuentos motores, danzas o teatro de sombras).

Conciencia y expresiones culturales
Desarrollo de tareas que favorezcan el conocimiento e interacción multicultural, como en los juegos o danzas populares.

Uso de actividades que busquen transmitir el conocimiento del patrimonio histórico-cultural de la motricidad humana (por ejemplo, las Olimpiadas).
Evaluación de comportamientos de riesgo para la salud pública vinculados a la actividad físico-deportiva y promoción de una vida activa y de calidad.

Uso de la evaluación como herramienta de aprendizaje para favorecer la autosuperación y perseverancia del alumnado.

Inclusión del alumno en la evaluación para valorar la pertinencia de sus decisiones e iniciativas (autoevaluación o coevaluación).

Se precisan futuras revisiones que profundicen en la relación entre los descriptores de esta competencia y la evaluación por competencias en EF. 
Además, es de interés apuntar que una adecuada enseñanza por competencias debe reunir unas características específicas, que van más allá de la planificación y análisis de los comportamientos asociados con cada una de las competencias clave. La Orden ECD/65/2015 prescribe la atención a la metodología y la evaluación como dos pilares para una respuesta educativa de calidad en un enfoque por competencias. Blázquez y Sebastiani (2009), Hong (2012) o Zapatero-Ayuso et al. (2012) apuntan a la necesidad de que la metodología esté centrada en el alumno y sea de carácter holístico, así como la necesidad de que la evaluación sea formativa y de carácter observable. En este sentido, la intervención debe promover metodologías activas, enfoques interdisciplinares y que favorezcan la puesta en práctica de los aprendizajes adquiridos y, al mismo tiempo, garantizar una evaluación objetiva, observable, continua, criterial y que use instrumentos específicos como rúbricas o portafolios.

A modo de limitación y prospectiva, es necesario puntualizar que este análisis ha supuesto una concreción de descriptores de carácter inductivo (Pérez-Pueyo, 2013), mediante el análisis de la normativa y la literatura de expertos, al mismo tiempo que se ha enriquecido con un proceso de verificación intersubjetiva entre los participantes en el trabajo. No obstante, siguiendo las orientaciones de Hong (2012), Hortigüela et al. (2015), Méndez-Alonso, Méndez-Giménez y Fernández-Río (2015) y Pérez-Pueyo (2013), parece necesario advertir que toda concreción del currículo competencial debe complementarse con una reflexión colaborativa y compartida en los centros educativos. Por tanto, puede ser una línea futura de desarrollo para este trabajo adoptar un carácter más holístico, incluyendo más docentes y coordinando este análisis conceptual con docentes de otras asignaturas. Ahora bien, este análisis puede ser una guía para el docente de EF, que impulse el trabajo competencial en la asignatura a través de la concreción de las competencias y el análisis de su relación con la dinámica habitual de sus clases. Con ello, se podría atender a las demandas del profesorado de EF, que declara una desinformación y falta de conocimiento sobre este elemento curricular (Barrachina y Blasco, 2012; Caballero, 2013; Zapatero-Ayuso et al., 2012). 


\section{REFERENCIAS}

Barrachina, J. y Blasco, J.E. (2012). «Análisis del desarrollo de las competencias básicas en el currículum de la Educación Física en la ESO en la Marina Baixa. Un estudio de caso». Apunts: Educación física y deportes, 110, 36-44.

Blázquez, D. y Sebastiani, E.M. (2009). Enseñar por competencias en Educación Física. Barcelona: INDE.

Bolívar, A. (2010). Competencias básicas y Currículum. Síntesis: Madrid.

Caballero, J.A. (2013). «La contribución del área de la Educación Física a las competencias básicas: opinión de los docentes». EmásF, Revista Digital de Educación Física, 4 (21), 1-18.

Calahorro, F., Lara, A. y Torres-Luque, G. (2010). «Competencias básicas en Educación Física. Identificación y Desarrollo». Arte y movimiento, 2, 31-39.

Cañabate, D., Zagalaz, M.L., Lara, A. y Cachón, J. (2011). «Aportaciones de la educación física al desarrollo de la competencia básica: autonomía e iniciativa personal». Tándem: Didáctica de la Educación Física, 11 (37), 69-77.

Consejo Europeo (2006). «Recomendación del parlamento europeo y del consejo de 18 de diciembre de 2006 sobre las competencias clave para el aprendizaje permanente». Diario Oficial de Europa, L394/10, de 30/12/2006, 10-18.

Contreras, O. y Cuevas, R. (2011). Las competencias básicas desde la Educación Física. Barcelona: INDE.

Decreto 23/2007, de 10 de mayo, del Consejo de Gobierno, por el que se establece para la Comunidad de Madrid el currículo de la Educación Secundaria Obligatoria. Boletín Oficial de la Comunidad de Madrid de 29 de mayo de 2007.

Decreto 57/2007, de 10 de mayo, por el que se establece el currículo de la Educación Secundaria Obligatoria en la Comunidad Autónoma de Cantabria. Boletín Oficial de Cantabria de 25 de mayo de 2007.

Decreto 69/2007, de 29 de mayo, por el que se establece y ordena el currículo de la Educación Secundaria Obligatoria en la Comunidad Autónoma De Castilla-La Mancha. Diario Oficial de Castilla-La Mancha de 1 de junio de 2007.

Decreto 74/2007, de 14 de junio, por el que se regula la ordenación y se establece el currículo de la Educación secundaria obligatoria en el Principado de Asturias. Boletín Oficial del Principado de Asturias de 12 de julio de 2007.

Decreto 89/2014, de 24 de julio, del Consejo de Gobierno, por el que se establece para la Comunidad de Madrid el Currículo de la Educación Primaria. Boletín Oficial de la Comunidad de Madrid de 25 de julio de 2014.

DeCreto 133/2007, de 5 de julio, por el que se regulan las enseñanzas de la educación secundaria obligatoria en la Comunidad Autónoma de Galicia. Boletín Oficial de Galicia de 13 de julio de 2007.

Decreto 175/2007, de 16 de octubre, por el que se establece el currículo de la Educación Básica y se implanta en la Comunidad Autónoma del País Vasco. Boletín Oficial del País Vasco de 13 de noviembre de 2007.

Delgado-Noguera, M.A. (1991). Los estilos de enseñanza en Educación Física. Granada: Universidad de Granada. 
Direction générale de l'enseignement scolaire (2006). «Décret du 11 de Julliet 2006. Le socle commun des connaissances et des compétences». París: Ministère Éducation Nationale Enseignement Supérier Recherche. Disponible en: http://media.education.gouv.fr/ file/51/3/3513.pdf.

Ellström, P.E. y Коск, H. (2008). «Competence Development in the Workplace: Concepts, Strategies and Effects». Asia Pacific Education Review, 9 (1), 5-20.

European Commission, EACEA y Eurydice (2012). Developing Key Competences at School in Europe: Challenges and Opportunities for Policy. Eurydice Report. Luxembourg: Publications Office of the European Union.

Giráldez, A. (2007). La competencia cultural y artística. Madrid: Alianza Editorial.

HonG, W.-P. (2012). «An international study of the changing nature and role of school curricula: from transmitting content knowledge to developing students' key competencies». Asia Pacific Education Review, 13 (1), 27-37.

Hortigüela, D., Abella, V. y Pérez-Pueyo, A. (2015). «¿Se han Implantado las competencias básicas en los centros educativos? Un estudio mixto sobre su programación como herramienta de aprendizaje». Revista Iberoamericana de Evaluación Educativa, 8 (1), 177-192.

ILLERIS, K. (2008). «Competence Development -the key to modern education, or just another buzzword?». Asia Pacific Education Review, 9 (1), 1-4.

Ley Orgánica 2/2006, de 3 de mayo, de Educación. Boletín Oficial del Estado de 4 de mayo de 2006.

Ley Orgánica 8/2013, de 9 de diciembre, para la mejora de la calidad educativa. Boletín Oficial del Estado de 10 de diciembre de 2013.

López-Pacheco, J. (2010). «Contribución de la Educación Física a la adquisición de las competencias básicas en Bachillerato». EmásF, Revista Digital de Educación Física, 1 (6), 36-46.

Martín, E. y Monereo, A. (2007). Competencia para aprender a aprender. Madrid: Alianza Editorial.

Martín, X. y Puig, J.M. (2007). Las siete competencias básicas para educar en valores. Barcelona: GRAO.

Martínez-López, F.J. (2009). «Las tecnologías de la información y la comunicación (TIC) y las competencias básicas en educación [en línea]». Espiral. Cuadernos del Profesorado, 2 (3), 15-26. Disponible en: http://www.cepcuevasolula.es/espiral.

Méndez-Giménez, A., Sierra, B. y Mañana, J. (2013). «Percepciones y creencias de los docentes de primaria del Principado de Asturias sobre las competencias básicas». Revista de Educación, 362, 737-761.

Méndez-Alonso, D., Méndez-Giménez, A. y Fernández-Río, F.J. (2015). «Análisis y valoración del proceso de incorporación de las Competencias Básicas en Educación Primaria». Revista de Investigación Educativa, 33 (1), 233-246.

Monarca, H. y Rappoport, S. (2013). «Investigación sobre los procesos de cambio educativo: El caso de las competencias básicas en España». Revista de Educación, Extraordinario, 54-78.

Monereo, C. (2005). «Internet, un espacio idóneo para desarrollar las competencias básicas». En C. Monereo (comp.), Internet y competencias básicas. Aprender a colaborar, a comunicarse, a participar y a aprender (pp. 5-26). Barcelona: GRAO.

Moya, J. (2008). De las competencias básicas al curriculum integrado. Disponible en: http://antonio-jimenez.com/documentos/Libros/Atlantida-Competencias-curriculo-integrado.pdf.

Moya, J. y Luengo, F. (2011). Teoría y práctica de las competencias básicas. Barcelona: GRAO. 
Muñoz-Díaz, J.C. (2010). «Las competencias básicas. Desarrollo a través de una unidad didáctica de Educación Física». EmásF, Revista Digital de Educación Física, 1 (3). Disponible en: http:// emasf.webcindario.com/NUEMRO_3_EMASF.pdf.

Gordon, J., Halász, G., Krawczyk, M., Leney, T., Michel, A., Pepper, D., Putkiewicz, E. y Wisniewski, J. (2009). Key Competences in Europe: Opening Doors for Lifelong Learns across the School Curriculum and Teacher Education. Warsaw: CASE (Center for Social and Economic Research).

Orden ECD/65/2015, de 21 de enero, por la que se describen las relaciones entre las competencias, los contenidos y los criterios de evaluación de la educación primaria, la educación secundaria obligatoria y el bachillerato. Boletín Oficial del Estado de 29 de enero de 2015.

Pérez-Pueyo, A. (2013). Programar y evaluar competencias básicas en 15 pasos. Barcelona: GRAO.

Pérez-Serrano, G. (1994). Investigación cualitativa. Retos e interrogantes. Vol II. Técnicas y análisis de datos. Madrid: La Muralla.

Quintero, L., Jiménez-Jiménez, F. y Area, M. (2016). «Las e-actividades: aplicaciones y recursos web». Tándem: Didáctica de la Educación Física, 53, 12-18.

Ramírez, A. (2015). «La formación del profesorado de Educación Primaria ante las competencias básicas». Revista Electrónica Interuniversitaria de Formación del Profesorado, 18 (3), 199-214.

Real Decreto 126/2014, de 28 de febrero, por el que se establece el currículo básico de la Educación Primaria. Boletín Oficial del Estado de 1 de marzo de 2014.

Real Decreto 1513/2006, de 7 de diciembre, por el que se establecen las enseñanzas mínimas de la Educación Primaria. Boletín Oficial del Estado de 8 de diciembre de 2006.

Real Decreto 1631/2006, de 29 de diciembre, por el que se establecen las enseñanzas mínimas correspondientes a la Educación Secundaria Obligatoria. Boletín Oficial del Estado de 5 de enero de 2007.

Rojano, D. (2010). «Uso de recursos TIC en la clase de Educación Física. Una experiencia positiva con el vídeo digital y el salto vertical». Retos. Nuevas tendencias en Educación Física, Deporte y Recreación, 17, 107-110.

Rychen, D. y SAlganik, L. (2004). Definir y seleccionar las competencias fundamentales para la vida. México D.F.: Fondo de Cultura Económica.

Salmerón, M. (2010a). «Descriptores de las competencias básicas en el área de Educación Física». Lecturas: Educación fisica y deportes, Revista Digital. 15 (149). Disponible en: http://www. efdeportes.com/efd149/descriptores-de-las-competencias-basicas-en-educacion-fisica.htm.

Salmerón, M. (2010b). «Programación de aula de Educación Física por competencias básicas en educación secundaria obligatoria». Lecturas: Educación física y deportes, 15 (143). Disponible en: http://www.efdeportes.com/efd143/competencias-basicas-en-educacion-secundaria-obligatoria.htm.

SAntos, M.L. y Martínez, L.F. (2011). «Aprendizaje integrado de las actividades en el medio natural desde las competencias en la ESO». Tándem: Didáctica de la Educación Física, 36, 53-60.

Sarramona, J. (2004). Las competencias básicas en la educación obligatoria. Barcelona: Ceac.

TeJADA, J. (2012). «La alternancia de contextos para la adquisición de competencias profesionales en escenarios complementarios de educación superior: marco y estrategia». Educación XXI, 15 (2), 19-40. 
Tiana, A., Moya, J., y Luengo, F. (2011). «Implementing Key Competences in Basic Education: reflections on curriculum design and development in Spain». European Journal of Education, 46 (3), 307-322.

Ureña, F. (2010). La Educación Física en Secundaria basada en competencias. Proyecto curricular y programación. Barcelona: INDE.

Zapatero-Ayuso, J.A., GonzÁlez-Rivera, M.D. y Campos-Izquierdo, A. (2012). «La formación de los docentes de Educación Física en torno a la enseñanza por competencias a través de un grupo de discusión». EmásF, Revista Digital de Educación Física, 3 (17), 1-15. 
